\title{
Comprensión lectora y metacognición: Análisis de las actividades de lectura en dos textos de estudio de la asignatura de Lenguaje y Comunicación de séptimo año básico
}

\author{
Reading comprehension and metacognition: Analysis of reading activities into two \\ textbooks of seventh year for the course Language and Communication \\ Compreensão de leitura e metacognição: Análise das atividades de leitura em dois \\ livros didáticos para o Curso de Língua e Comunicação Básica sétimo ano
}

\author{
Ramírez Peña, Pamela ${ }^{a}$; Rossel Ramírez, Katherine ${ }^{b}$; Nazar Carter, Gabriela ${ }^{c}$ \\ aDepartamento de Español, Universidad de Concepción, Concepción, Chile. \\ Teléfono: 41-2204123. \\ Correo electrónico: pamerami@udec.cl \\ ${ }^{\text {b} U n i v e r s i d a d ~ d e ~ C o n c e p c i o ́ n, ~ C o n c e p c i o ́ n, ~ C h i l e . ~}$ \\ Teléfono: 41-2654538. \\ Correo electrónico: krossel@udec.cl \\ 'Departamento de Psicología, Universidad de Concepción, Concepción, Chile. \\ Teléfono: 41-2203946. \\ Correo electrónico: gnazar@udec.cl
}

\begin{abstract}
RESUMEN
La investigación que se presenta a continuación corresponde a un estudio cualitativo de análisis documental cuyo objetivo fue conocer el uso de estrategias metacognitivas en las actividades de comprensión lectora en dos textos escolares de séptimo año básico. El análisis se realizó en función de los momentos de la lectura propuestos en el enfoque metacognitivo de la comprensión de textos. Los resultados muestran que en ambos libros las actividades de lectura se concentran principalmente en el momento Después de la lectura, con predominio de la estrategia Contestar preguntas. El momento Durante la lectura presenta el menor porcentaje de actividades propuestas en ambos libros. Se concluye que la didáctica de la comprensión lectora en estos textos no considera el desarrollo de estrategias metacognitivas orientadas a formar lectores estratégicos, puesto que las actividades se focalizan en el resultado más que en el proceso de lectura.
\end{abstract}

Palabras clave: comprensión lectora, metacognición, textos de estudio, estrategias de lectura, séptimo año básico.

\begin{abstract}
The research presented below is a qualitative study of a documentary analysis which aim was to better understand the use of metacognitive strategies in reading comprehension activities of two elementary seventh grade textbooks. The analysis performed was based on the moments of reading proposed in the metacognitive approach for reading comprehension. The results show that in both books reading activities are mainly concentrated in the After reading time, predominating the strategy of Answering questions. During reading time has the lowest percentage of activities proposed in both textbooks. It is concluded that the didactic of reading comprehension in these texts does not consider the development of metacognitive strategies leading to form strategic readers since the activities are focused on the outcome rather than on the process of reading.
\end{abstract}

Key words: reading comprehension, metacognition, seventh year textbooks, reading strategies. 


\section{RESUMO}

A pesquisa corresponde a um estudo qualitativo de análise documental que objetivou conhecer o uso de estratégias metacognitivas nas atividades de compreensão de leitura de dois textos didáticos do sétimo ano básico. A análise foi realizada em função dos momentos da leitura propostos no enfoque metacognitivo da compreensão de textos. Resultados mostram que nos dois textos as atividades de leitura se concentram principalmente no momento do Pós-leitura, com predomínio na estratégia de Responder preguntas. O momento "durante a leitura" representa a menor percentagem de atividades propostas em ambos os textos. Conclui-se que a didática da compreensão de leitura em ambos os textos desconsidera o desenvolvimento de estratégias metacognitivas orientadas a formar leitores estratégicos já que as atividades se focalizam mais no resultado do que no processo de leitura. básico.

Palavras chave: compreensão de leitura, metacognição, textos didáticos, estratégias de leitura, sétimo ano

\section{INTRODUCCIÓN}

El déficit en comprensión lectora en los estudiantes chilenos representa un gran desafío para el sistema educacional de nuestro país. Numerosas investigaciones (Mcnamara, 2004; López y Arcienagas, 2013) sostienen que mejorar los niveles de comprensión textual supone un proceso consciente y controlado de la actividad de lectura, por lo que el enfoque metacognitivo puede contribuir positivamente a mejorar los niveles de comprensión lectora.

Estudios recientes (Karbalaei, 2011; Pihlainen \& Keinonen, 2011; Ozuru, Kurby \& McNamara, 2012; Yang, 2012; Mason, Meadan-Kaplansky, Hedin \& Taft, 2013) enfatizan la necesidad de profundizar en la relación entre la metacognición y la comprensión lectora, sin embargo, la investigación en el ámbito escolar es escasa. Al respecto, Ray (2011) realiza una investigación con textos escolares de ciencias y analiza las actividades de comprensión de textos expositivos en textos escolares de $2^{\circ}, 3^{\circ}$ y $4^{\circ}$ grado, concluyendo que tanto los libros para el profesor como para los estudiantes presentan carencias en la promoción de la comprensión de textos expositivos.

Considerando lo anterior esta investigación buscó conocer en qué medida el enfoque didáctico subyacente a las actividades de comprensión lectora de dos textos escolares promueve el uso de estrategias metacognitivas. Para responder a este cuestionamiento se analizaron las actividades de lectura que se encuentran en dos textos de estudio de séptimo año básico de la asignatura Lenguaje y Comunicación elaborados por la editorial Santillana.

El objetivo general de esta investigación fue identificar el uso de estrategias metacognitivas en el enfoque didáctico que sustenta las actividades de comprensión lectora de dos textos de Lenguaje y Comunicación de séptimo año básico. Como objetivos específicos se plantearon a) determinar la medida en que las actividades expuestas en ambos libros estimulan la planificación, supervisión y evaluación del proceso de lectura; y b) identificar la existencia de diferencias en el enfoque didáctico de la comprensión lectora entre ambos textos de estudio. De esta forma, el análisis de los libros seleccionados dará luces respecto de la forma en que se trabaja la didáctica de la comprensión lectora en establecimientos municipales, particulares subvencionados y particulares pagados de Chile. 


\section{MARCO TÉORICO}

\subsection{LA COMPRENSIÓN LECTORA COMO PROCESO DINÁMICO E INTERACTIVO}

El estudio realizado se sustenta en la teoría de la construcción-integración (Kintsch, 1988, 1998), la que postula que quien comprende procesa el texto por partes (cláusulas, oraciones) y el procesamiento de cada una de estas partes incluye dos fases: una de construcción y otra de integración. Durante la fase de construcción se llevan a cabo los procesos de menor nivel (decodificación, recuperación de palabras, análisis gramatical) y se produce un cambio de formato de la información, ya que lo que se encuentra escrito en el texto se traduce a un formato proposicional para ser representado en la mente del lector. Estas proposiciones se organizan en una red de coherencia que contiene tanto asociaciones inmediatas a las ideas del texto como inferencias que contribuyen a la coherencia del texto y generalizaciones del mismo.

Durante la fase de integración es posible postular la participación de mecanismos inhibitorios que permiten mantener activa la red de proposiciones (red de coherencia) en que existe una mayor interconexión desactivando los conceptos inapropiados contextualmente. De esta forma, al final de cada ciclo de construcción-integración un pequeño número de elementos altamente activos de la cláusula analizada se mantienen por sobre la capacidad limitada de la memoria temporal (Baddeley, 1986) para un procesamiento posterior (Kintsch \& Van Dijk, 1978). Gracias a este traspaso de elementos desde la memoria temporal o de trabajo a la memoria a largo plazo es posible construir una representación textual coherente o, dicho de otro modo, una representación semántica del texto que se codifica en la memoria de largo plazo (MLP) en distintos niveles.

Kintsch (1988; Ericsson \& Kintsch, 1995) propone tres niveles de representación en la construcción de la representación semántica del texto. En primer lugar, el nivel de representación superficial, el que se elabora a partir de la información lingüística que aporta el texto y constituye un breve registro de la redacción del texto en la memoria. En segundo lugar, el nivel de representación del texto base, que especifica las relaciones semánticas entre los distintos componentes y partes del texto mediante un formato proposicional. Finalmente, el nivel de representación del modelo de situación, que corresponde a un modelo mental -una suerte de representación cognitiva análoga no proposicional- de la situación referida en el texto.

Respecto de la importancia de cada uno de estos niveles para el proceso de comprensión, los hallazgos obtenidos por Kintsch, Welsch, Schmalhofer \& Zimny (1990) señalan que cada uno de estos niveles desempeña un papel diferencial en la comprensión, memoria y, por ende, aprendizaje a partir de un texto. Al respecto, Gárate, Gutiérrez, Luque, García y Elosúa (1999) señalan que el nivel superficial del texto no parece jugar un papel importante para la memoria y aprendizaje, ya que su función estaría más relacionada con las operaciones de reconocimiento de palabras necesarias para procesos posteriores. Con relación al nivel de representación del texto base, señalan que este hace posible establecer una jerarquía de ideas provenientes del texto debido a que permite conectar ideas que provienen de distintas partes del mismo. Finalmente, el nivel de representación del modelo situacional es, al parecer, el que mayor importancia tiene para el proceso de comprensión y aprendizaje a partir de un texto, toda vez que en este nivel se generan inferencias y oraciones nuevas que en conjunto constituyen una representación de la situación que el 
texto describe y pueden soportar reelaboraciones que permitan desarrollar operaciones destinadas al aprendizaje.

\subsection{METACOGNICIÓN}

Según Flavell (1976, Cit. en Osses y Jaramillo, 2008: 191), la metacognición es el conocimiento que cada persona tiene respecto de sus propios procesos y productos cognitivos o algún aspecto que se relacione con ellos. Es entendida también como la supervisión constante de la regulación y organización de estos procesos, que se relacionan con asuntos cognitivos sobre los que actúa en búsqueda de una meta u objetivo determinado. En los últimos años este término ha sido comprendido como el conocimiento que las personas tienen acerca de su funcionamiento cognitivo.

Uno de los modelos más importantes que estudia la metacognición es el planteado por Flavell en 1985 (Cit. en Lacon y Ortega, 2008:8), el que propone dos dominios metacognitivos: el del conocimiento metacognitivo y el de la experiencia metacognitiva. El primero alude a los conocimientos que tienen las personas sobre la cognición, en relación con tareas y concernientes a estrategias. Relativo al segundo dominio metacognitivo, las experiencias metacognitivas se definen como las sensaciones que experimenta conscientemente un individuo que está llevando a cabo un proceso cognitivo: darse cuenta de la dificultad de la tarea que se está realizando, o tener la sensación de que el camino elegido para resolverla es inadecuado, o sentir que está alcanzando su objetivo.

Además, la metacognición contribuye al desarrollo de los conocimientos tanto declarativos como los procedimentales y se va fortaleciendo y acumulando con los años, integrándose a un conjunto de conocimientos metacognitivos que cada persona desarrolla en su vida. Según Flavell, parece posible que los conocimientos y las vivencias cognitivas y metacognitivas estén continuamente formándose e interactuando en cualquier actividad cognitiva (Lacon y Ortega, 2008).

\subsection{COMPRENSIÓN LECTORA Y METACOGNICIÓN: USO DE ESTRATEGIAS}

Considerando el modelo que explica el proceso de comprensión (teoría de construcciónintegración) es posible plantear que la comprensión de un texto solo se puede lograr toda vez que el sujeto decide llevar a cabo un proceso consciente e intencionado, con el fin de construir el modelo de situación que plantea el texto (Kintsch, 1998). Es por ello que el rol de la metacognición en las tareas de comprensión lectora es fundamental, ya que el lector debe desarrollar un comportamiento estratégico en función de un objetivo específico (López y Arcienagas, 2004).

López y Arcienagas (2004) plantean que las estrategias son actividades intencionales y deliberadas, en donde se involucra conscientemente el sujeto para lograr las metas que se ha propuesto. En el ámbito de la comprensión lectora dichas metas serán alcanzadas por acciones específicas encaminadas al procesamiento y la comprensión adecuada del texto. En términos generales, estas estrategias se dividen en estrategias cognitivas y estrategias metacognitivas. Las primeras corresponden a un conjunto de actividades y procesos mentales que los aprendices realizan de manera consciente o inconsciente. Por ejemplo, en el caso de la lectura, establecer un propósito de lectura; 
acceso al conocimiento previo y uso de los esquemas relevantes; y uso adecuado de las claves del texto. Las estrategias metacognitivas, en cambio, son aquellas que permiten al sujeto controlar, supervisar y evaluar el proceso de lectura. Estas estrategias se centran en tres aspectos básicos:

1. Adquisición o refinamiento del conocimiento en el proceso de lectura y de comprensión de textos.

2. Toma de conciencia de la necesidad de controlar y de regular el propio proceso y, en consecuencia, de asumirlo como tal.

3. Mayor motivación, responsabilidad y desarrollo de una actitud positiva como lector activo, reflexivo, crítico y, por lo tanto, aprendiz autónomo y responsable.

Tanto el uso de estrategias cognitivas como metacognitivas determinan el proceso de metacomprensión de un texto, cuyos objetivos básicos se resumen en:

a) La toma de conciencia de la lectura como proceso estratégico y de sus implicaciones en los procesos de comprensión de textos y aprendizaje a partir de ellos.

b) El autocontrol y la autorregulación del proceso durante el mismo y su evaluación después de su ejecución. (López y Arcienagas, 2004).

López y Arcienagas (2003) plantean que el uso de estrategias orientadas a la metacomprensión está determinado por el momento de la lectura en el cual se llevan a cabo. Es por ello que, desde el punto de vista de la metacognición, se ha dividido el proceso de lectura de un texto escrito en tres momentos, cada uno de ellos asociado a estrategias específicas, como se señala a continuación.

Antes de la lectura: a esta etapa se le denomina planificación, ya que el lector debe precisar los propósitos de lectura y las estrategias que utilizará para conseguirlos.

Durante la lectura: corresponde a la etapa de supervisión. Por ello, las estrategias que se emplean en esta etapa están orientadas a controlar la calidad del proceso que se está desarrollando. Actividades como tomar notas, subrayar, hacer preguntas al texto, releer selectivamente el texto, entre otras, son estrategias orientadas a la supervisión del proceso.

Después de la lectura: corresponde a la etapa de evaluación. Estas estrategias tienen como finalidad determinar los logros obtenidos en relación con los objetivos establecidos en la etapa de planificación. Por ejemplo, elaborar un resumen, realizar organizadores gráficos que representen el contenido del texto, consultar otras fuentes, contestar preguntas, entre otras; son estrategias orientadas a evaluar la calidad de la lectura en función de los objetivos propuestos.

La revisión teórica expuesta en las líneas precedentes y las investigaciones antes citadas demuestran que la comprensión lectora, desde una perspectiva didáctica, es una actividad que implica un enfoque estratégico, donde la metacognición tiene un rol relevante.

El análisis que se presenta a continuación pretende conocer de qué forma se aborda la didáctica de la comprensión lectora en textos escolares chilenos indagando en la relación que se establece entre las tareas de comprensión lectora y la metacognición. 


\section{MÉTODO}

Se realizó un estudio cualitativo de análisis documental cuya finalidad fue conocer el uso de estrategias metacognitivas en la didáctica de la comprensión lectora de dos textos escolares de séptimo año básico de la editorial Santillana. Ambos textos corresponden a la última versión del libro elaborada el año 2009, uno de ellos (libro 1) es distribuido por el Ministerio de Educación (MINEDUC) a establecimientos municipales y particulares subvencionados, el libro 2 es utilizado en establecimientos particulares pagados. La selección de textos se realizó a base de la frecuencia de uso y la etapa de desarrollo metacognitivo de los estudiantes. Respecto del primer criterio, Vieiro y Gómez (2004) destacan los aportes de Piaget relativos al desarrollo cognitivo de los niños, quien sostiene que a partir de los 11-12 años el sujeto es capaz de realizar abstracciones y reflexionar en torno a sus propios procesos de pensamiento. En esta misma línea, Flavell (1976) sostiene que el conocimiento metacognitivo y la capacidad de regulación de cualquier tarea cognitiva tiene una tendencia evolutiva propia de la tercera infancia y adolescencia. Concerniente al segundo criterio, tras la revisión de las listas de útiles escolares propuestas en colegios particulares pagados de Chile fue posible constatar que en séptimo año básico el libro de Lenguaje y Comunicación solicitado pertenece, en su mayoría, a la editorial Santillana, la que a su vez ha elaborado el libro que distribuye el MINEDUC.

En los libros de séptimo año básico de Lenguaje y Comunicación se contemplan actividades asociadas al contenido de la asignatura (conocimiento del lenguaje, literatura y medios masivos de comunicación) distribuidas en 7 unidades de trabajo, en el caso del libro 1, y 8 unidades de trabajo en el libro 2. El universo utilizado para el análisis corresponde a las actividades que se proponen a partir de las lecturas contempladas en ambos libros de Lenguaje y Comunicación de séptimo año básico. De este universo se analizaron todas las actividades de compresión lectora: 229 en total, para el libro 1; y, 302 en total para el libro 2. Se excluyeron actividades como herramientas de la lengua, evaluaciones de proceso, evaluación de la unidad y las actividades que introducen el contenido central de cada unidad por no estar centradas en el proceso didáctico de la comprensión lectora.

Para indagar acerca de dichas actividades se realizó un análisis de contenido que se estructuró basado el el modelo planteado por López y Arcienagas (2004), que divide el proceso de comprensión lectora en tres momentos (antes, durante y después) y asocia a cada momento a una etapa particular del proceso (etapa de planificación, etapa de supervisión y etapa de evaluación. Se elaboró un esquema de codificación sobre la base de la literatura disponible, el que fue progresivamente refinado con la opinión de dos expertos. Este esquema fue validado tras la revisión de las actividades de comprensión lectora propuestas en un texto de octavo año básico de la asignatura de Lenguaje y Comunicación.

En función de lo anterior se establecieron las siguientes categorías de análisis: 
Tabla 1. Estrategias utilizadas en la comprensión de textos y su esquema de codificación

\begin{tabular}{|l|l|l|}
\hline \multicolumn{1}{|c|}{ Antes de la lectura } & \multicolumn{1}{|c|}{ Durante la lectura } & \multicolumn{1}{c|}{ Después de la lectura } \\
\hline Etapa de planificación & Etapa de supervisión & Etapa de evaluación \\
\hline Establecer una meta de lectura (M) & Comprobar hipótesis (CH) & $\begin{array}{l}\text { Contestar preguntas de compren- } \\
\text { sión lectora (CP) }\end{array}$ \\
\hline $\begin{array}{l}\text { Hojear, observar el texto a fin de reconocer } \\
\text { la estructura (O) }\end{array}$ & $\begin{array}{l}\text { Realizar/contestar preguntas } \\
\text { a medida que se avanza en } \\
\text { la lectura (CP) }\end{array}$ & $\begin{array}{l}\text { Resumir, esquematizar, construir } \\
\text { mapas conceptuales a partir del } \\
\text { contenido del texto (R/E/M) }\end{array}$ \\
\hline Definir una estrategia de lectura (E) & $\begin{array}{l}\text { Subrayar conceptos clave o o } \\
\text { palabras desconocidas (S) }\end{array}$ & $\begin{array}{l}\text { Examinar, revisar y evaluar la } \\
\text { estrategia empleada en función } \\
\text { del objetivo de lectura (EE) }\end{array}$ \\
\hline $\begin{array}{l}\text { Aclarar conceptos que podrían aparecer en } \\
\text { el texto según la estructura o el título del } \\
\text { texto (AC) }\end{array}$ & $\begin{array}{l}\text { Usar el contexto para cons- } \\
\text { truir el significado de las } \\
\text { palabras desconocidas (VC) }\end{array}$ & \\
\hline $\begin{array}{l}\text { Establecer hipótesis o predicciones respecto } \\
\text { al contenido del texto (H/P) }\end{array}$ & & \\
\hline Activación de conocimientos previos (ACP) & & \\
\hline
\end{tabular}

En términos generales, las actividades de comprensión lectora se plantean como preguntas o instrucciones en cada momento de la lectura. A continuación se presentan algunos ejemplos de actividades según las categorías de análisis (Tabla 2).

Tabla 2. Ejemplos de actividades de comprensión lectora asociadas a cada una de las estrategias utilizadas en las categorías de análisis

\begin{tabular}{|l|l|l|l|l|l|}
\hline \multicolumn{1}{|c|}{$\begin{array}{c}\text { Antes } \\
\text { de la lectura }\end{array}$} & \multicolumn{1}{|c|}{ Ejemplo } & $\begin{array}{c}\text { Durante la } \\
\text { lectura }\end{array}$ & \multicolumn{1}{c|}{ Ejemplo } & \multicolumn{1}{c|}{$\begin{array}{c}\text { Después } \\
\text { de la lectura }\end{array}$} & \multicolumn{1}{c|}{ Ejemplo } \\
\hline $\begin{array}{l}\text { Establecer una } \\
\text { meta de lectura } \\
(\mathrm{M})\end{array}$ & $\begin{array}{l}\text { No se registran } \\
\text { actividades en } \\
\text { ambos libros }\end{array}$ & $\begin{array}{l}\text { Comprobar } \\
\text { hipótesis }(\mathrm{CH})\end{array}$ & $\begin{array}{l}\text { ¿Se demuestra en } \\
\text { los versos subraya- } \\
\text { dos que la canción } \\
\text { alude a quien } \\
\text { habías pensado? }\end{array}$ & $\begin{array}{l}\text { Contestar } \\
\text { preguntas de } \\
\text { comprensión } \\
\text { lectora }(\mathrm{CP})\end{array}$ & $\begin{array}{l}\text { ¿Qué razones tenían } \\
\text { los niños del colegio } \\
\text { para rechazar a } \\
\text { Simón? }\end{array}$ \\
\hline $\begin{array}{l}\text { Hojear, observar } \\
\text { el texto a fin } \\
\text { de reconocer la } \\
\text { estructura }(\mathrm{O})\end{array}$ & $\begin{array}{l}\text { ¿Cómo podrías } \\
\text { saber que el } \\
\text { texto que leerás } \\
\text { a continuación es } \\
\text { una noticia? }\end{array}$ & $\begin{array}{l}\text { Realizar/con- } \\
\text { testar preguntas } \\
\text { a medida que } \\
\text { se avanza en la } \\
\text { lectura }(\mathrm{CP})\end{array}$ & $\begin{array}{l}\text { ¿Qué diferen- } \\
\text { cias tendría esta } \\
\text { situación en la } \\
\text { actualidad? ¿O no } \\
\text { la habría? (pregun- } \\
\text { ta guiada entre la } \\
\text { lectura) }\end{array}$ & $\begin{array}{l}\text { Resumir, esque- } \\
\text { matizar, construir } \\
\text { mapas concep- } \\
\text { tuales a partir } \\
\text { del contenido del } \\
\text { texto (R/E/M) }\end{array}$ & $\begin{array}{l}\text { Haz un breve resu- } \\
\text { incorporando al menos de las palabras } \\
\text { cinco dia } \\
\text { destacadas en el texto. } \\
\text { Al escribir, reempláza- } \\
\text { las por un sinónimo }\end{array}$ \\
\hline
\end{tabular}




\begin{tabular}{|c|c|c|c|c|c|}
\hline $\begin{array}{c}\text { Antes } \\
\text { de la lectura }\end{array}$ & Ejemplo & $\begin{array}{c}\text { Durante la } \\
\text { lectura }\end{array}$ & Ejemplo & $\begin{array}{l}\text { Después } \\
\text { de la lectura }\end{array}$ & Ejemplo \\
\hline $\begin{array}{l}\text { Definir una } \\
\text { estrategia de } \\
\text { lectura (E) }\end{array}$ & $\begin{array}{l}\text { Lee el siguiente } \\
\text { texto aplicando } \\
\text { la técnica del } \\
\text { subrayado }\end{array}$ & $\begin{array}{l}\text { Subrayar con- } \\
\text { ceptos clave y/o } \\
\text { palabras desco- } \\
\text { nocidas (S) }\end{array}$ & $\begin{array}{l}\text { Lee atentamente } \\
\text { esta noticia y } \\
\text { anota en tu cua- } \\
\text { derno las palabras } \\
\text { que desconozcas }\end{array}$ & $\begin{array}{l}\text { Examinar, revisar } \\
\text { y evaluar la es- } \\
\text { trategia empleada } \\
\text { en función del } \\
\text { objetivo de lectu- } \\
\text { ra (EE) }\end{array}$ & $\begin{array}{l}\text { Intercambia tu mapa } \\
\text { conceptual con el de } \\
\text { un compañero. Pídele } \\
\text { que lo evalúe conside- } \\
\text { rando que: } \\
\text { a) contenga toda } \\
\text { la información } \\
\text { importante. } \\
\text { b) los conceptos estén } \\
\text { apropiadamente } \\
\text { relacionados }\end{array}$ \\
\hline $\begin{array}{l}\text { Aclarar concep- } \\
\text { tos que podrían } \\
\text { aparecer en el } \\
\text { texto según la } \\
\text { estructura o al } \\
\text { título del texto } \\
\text { (AC) }\end{array}$ & $\begin{array}{l}\text { ¿Qué entiendes } \\
\text { por familia? }\end{array}$ & $\begin{array}{l}\text { Usar el contexto } \\
\text { para construir } \\
\text { el significado } \\
\text { de las palabras } \\
\text { desconocidas } \\
\text { (VC) }\end{array}$ & $\begin{array}{l}\text { Completa este } \\
\text { cuadro siguiendo } \\
\text { el orden de iz- } \\
\text { quierda a derecha. } \\
\text { Las palabras que } \\
\text { no conozco. } \\
\text { Defino las palabras } \\
\text { según su contexto. } \\
\text { Busco definición } \\
\text { precisa en el } \\
\text { diccionario }\end{array}$ & & \\
\hline $\begin{array}{l}\text { Establecer } \\
\text { hipótesis y/o } \\
\text { predicciones } \\
\text { respecto al } \\
\text { contenido del } \\
\text { texto }(\mathrm{H} / \mathrm{P})\end{array}$ & $\begin{array}{l}\text { ¿Quién crees que } \\
\text { será el protago- } \\
\text { nista o personaje } \\
\text { principal de esta } \\
\text { narración: Simón } \\
\text { o su papá? ¿en } \\
\text { qué te basas para } \\
\text { afirmarlo? }\end{array}$ & & & & \\
\hline $\begin{array}{l}\text { Activación de } \\
\text { conocimientos } \\
\text { previos (ACP) }\end{array}$ & $\begin{array}{l}\text { ¿Qué reglamen- } \\
\text { tos conoces? }\end{array}$ & & & & \\
\hline
\end{tabular}

El análisis se inició con la clasificación de textos por cada libro en función de dos categorías: textos literarios y textos no literarios. Se entiende como texto literario aquel cuya intención comunicativa tiene una finalidad estética, aludiendo a un mundo ficticio. Los textos no literarios, en cambio, son aquellos en los cuales se refleja la realidad de alguna manera; son funcionales, pues en una sociedad alfabetizada se leen instrucciones, normas, noticias, etc. Las intenciones comunicativas que predominan en los textos no literarios son informar, convencer y persuadir. A continuación se presenta una Tabla en la cual se señalan los tipos de texto que se encontraron en ambos libros (Tabla 3). 
Tabla 3. Tipos de textos por cada clasificación

\begin{tabular}{|l|l|}
\hline Textos literarios & Textos no literarios \\
\hline Narraciones & Noticias \\
\hline Poemas & Entrevistas \\
Antipoemas & Afiches \\
Poesía visual & Infografía \\
\hline Obras dramáticas & Carta \\
\hline & Discurso público \\
& Textos normativos \\
\hline
\end{tabular}

Una vez clasificados, los tipos de texto fueron codificados utilizando el esquema de codificación presentado previamente. La codificación fue realizada por las investigadoras de manera independiente y se resolvieron en conjunto las diferencias en el uso de criterios de codificación. Posteriormente se sometió la codificación a juicio de expertos (profesoras de la asignatura de Lenguaje y Comunicación en ejercicio).

El proceso de codificación siguió el siguiente proceso de análisis:

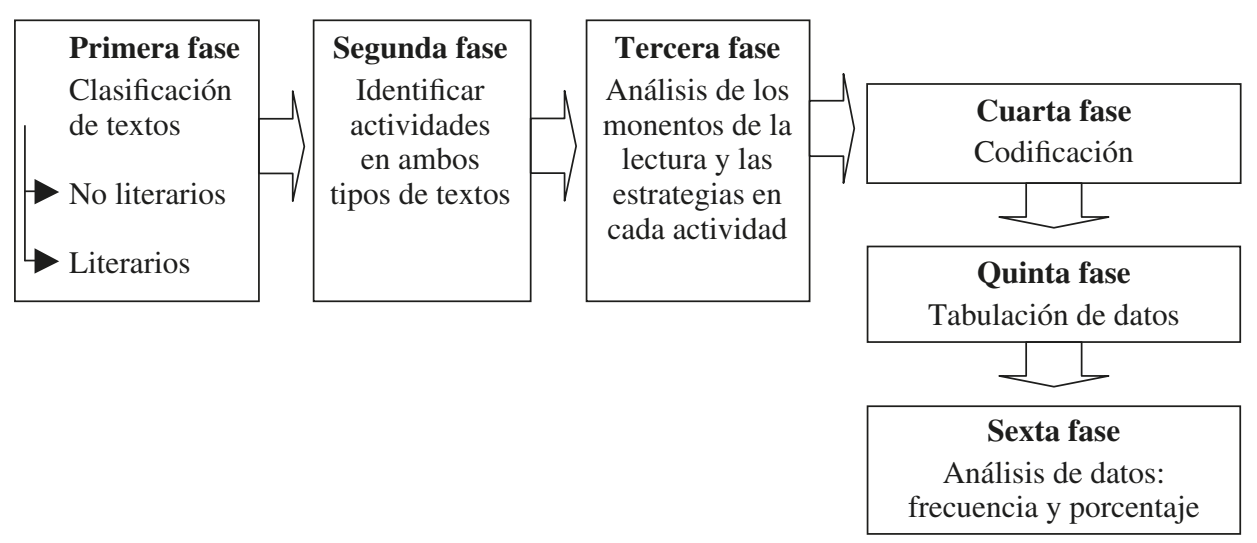

\section{RESULTADOS}

En el libro 1 se proponen 229 actividades de comprensión lectora; en el libro 2, 302 actividades. Al revisar la frecuencia y porcentajes de actividades por cada etapa de lectura se encontró que el libro 1 (Gráfico 1) en la etapa de planificación propone 48 actividades (21\% del total de actividades); en la etapa de supervisión se observan 31 actividades (13\% del total de actividades); y en la etapa de evaluación 150 actividades (65\% del total). En el libro 2 (Gráfico 1), en la etapa de planificación se proponen 53 actividades (17,5\%); en la etapa de supervisión 48 actividades (15,9\%); y en la etapa de evaluación 201 actividades $(65,6 \%)$. 
Gráfico 1. Porcentaje de actividades por etapa de la lectura en el libro 1 y libro 2

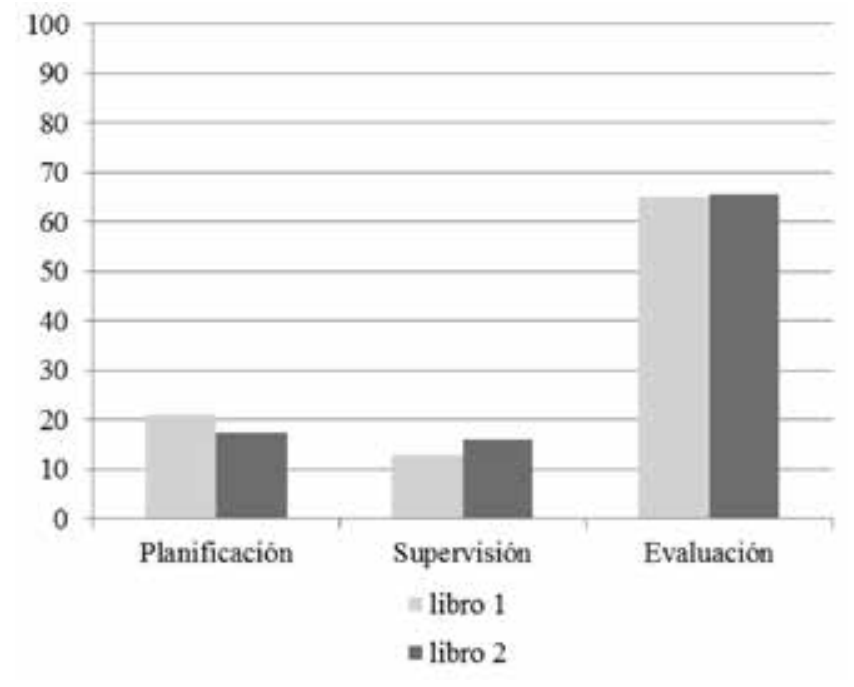

En ambos libros se observa que las actividades se concentran principalmente en la etapa de evaluación y en el momento Después de la lectura, cuyo objetivo es la evaluación de la comprensión y del proceso de lectura (65\% de las actividades en ambos libros). Por otro lado, es posible observar que en el momento Durante la lectura, etapa denominada supervisión, se observa el menor porcentaje de actividades propuestas en ambos libros.

\subsection{MOMENTO ANTES DE LA LECTURA}

Libro 1: en cuanto a los resultados obtenidos respecto de las actividades de comprensión lectora que utilizan una de las estrategias identificadas en el enfoque metacognitivo de la comprensión lectora, se observa que en el libro 1 el momento Antes de la lectura (etapa de planificación), el establecimiento de metas de la lectura (M) no contempla ninguna actividad. Respecto de la estrategia de observación del texto $(\mathrm{O})$, se identificaron seis actividades (2,6\% del total de actividades). En cuanto a determinar las estrategias de lectura (E), una actividad contempla este tipo de estrategia $(0,4 \%)$. Otra estrategia identificada en esta etapa de la lectura es reconocer/aclarar conceptos (AC), donde 14 actividades de comprensión lectora la utilizan $(6,1 \%)$. En la estrategia establecer hipótesis o predicciones antes de la lectura $(\mathrm{H} / \mathrm{P})$ se identificaron 15 actividades $(6,5 \%) \mathrm{y}$, por último, la activación de conocimientos previos (ACP), donde 12 actividades pretenden desarrollar esta estrategia $(5,2 \%)$.

Libro 2: al igual que en el libro 1, en el libro 2 no se identificaron actividades que usen la estrategia establecimiento de una meta de lectura (M). Lo mismo sucede con las estrategias observación del texto $(\mathrm{O})$ y determinación de una estrategia de lectura $(\mathrm{E})$. En cuanto a la estrategia reconocer/aclarar conceptos (AC), pertenecientes a esta misma 
etapa, tres actividades de comprensión lectora la utilizan (1\% del total de actividades). $\mathrm{Al}$ considerar la estrategia establecer hipótesis o predicciones antes de la lectura $(\mathrm{H})$, se identificaron 25 actividades $(8,3 \%) \mathrm{y}$, por último, la estrategia activación de conocimientos previos (ACP), se observa en 25 actividades del texto de estudio (8,3\%). A continuación se presentan los datos de cada libro (Gráfico 2)

Gráfico 2. Frecuencia de uso de estrategias metacognitivas en el momento Antes de la lectura en ambos libros

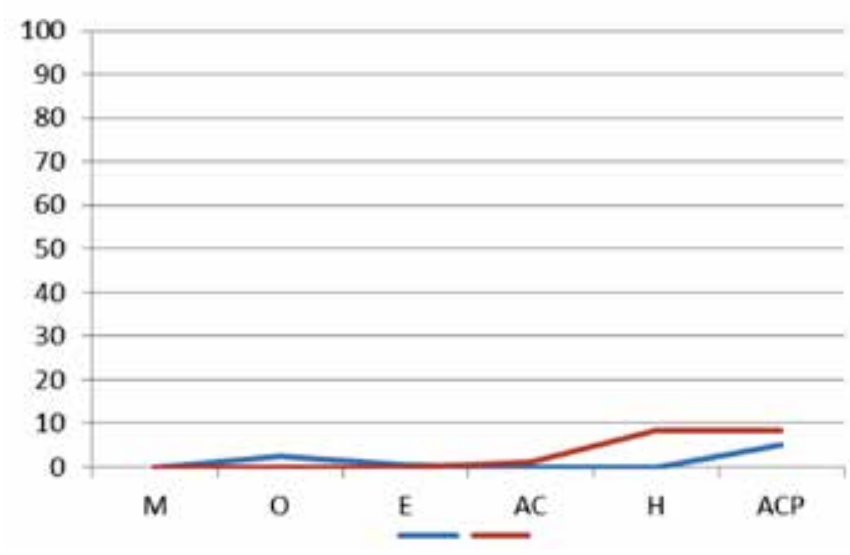

\subsection{MOMENTO DURANTE LA LECTURA}

Libro 1: en el momento Durante la lectura (etapa de supervisión), en el libro 1 se identificó una actividad orientada a la comprobación de las hipótesis $(\mathrm{CH})(0,4 \%)$. La estrategia responder preguntas (RP) se observa en 28 actividades (12,1\%). En cuanto a la estrategia de subrayado (S), se observa una actividad $(0,4 \%)$ al igual que la presencia de la estrategia de vocabulario contextual (VC) (una actividad, $0,4 \%$ del total de actividades).

Libro 2: se observó que el total de actividades de la etapa es representada por la estrategia contestar preguntas (CP) (15.9\% del total de actividades), vale decir, que no se identificaron actividades que utilizaran las estrategias comprobación de hipótesis $(\mathrm{CH})$, subrayado(S) y vocabulario contextual (VC) (Gráfico 3).

\subsection{MOMENTO DESPUÉS DE LA LECTURA}

Libro 1: las actividades orientadas a responder preguntas son $133(57,8 \%$ del total de actividades). En cuanto al uso de resúmenes/esquemas/mapas conceptuales (R/E/M), 16 actividades se relacionan con una de estas estrategias (6,9\% del total de actividades). Por último, en esta etapa una actividad se orienta al uso de evaluación de la estrategia utilizada (EE) (0,4\% del total de actividades). En el libro 2, 201 actividades representan la estrategia contestar preguntas $(\mathrm{CP})(64,6 \%)$. Finalmente, en las demás estrategias se observan tres 
Gráfico 3. Estrategias asociadas al momento durante la lectura en ambos libros

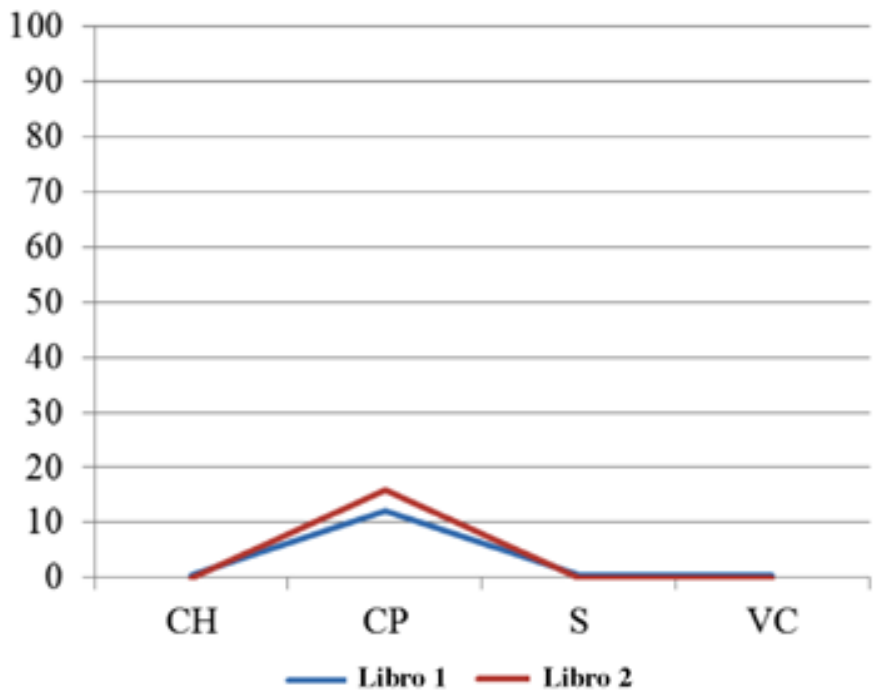

actividades que hacen uso de resúmenes/esquemas/mapas conceptuales (R/E/M) que corresponden al $1 \%$ del total de actividades y, por último, tres actividades se orientan a evaluar la estrategia utilizada (EE), lo que implica 1\% de las actividades (Gráfico 4).

Gráfico 4. Estrategias asociadas al momento después de la lectura en ambos libros

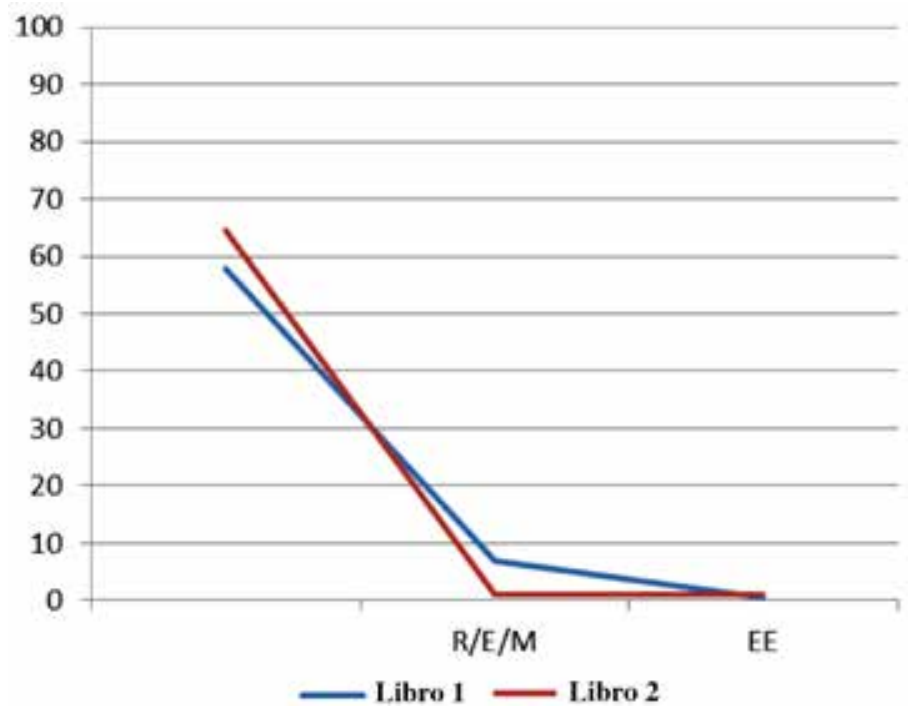

Los resultados obtenidos se muestran en la Tabla 4, que resume las frecuencias y porcentajes. 
Tabla 4. Frecuencias y porcentajes respecto del total de las actividades de comprensión lectora

\begin{tabular}{|l|l|c|c|c|c|}
\hline \multirow{2}{*}{$\begin{array}{c}\text { Etapa de } \\
\text { lectura }\end{array}$} & \multicolumn{1}{c|}{ Estrategias } & \multicolumn{2}{c|}{ Frecuencias } & \multicolumn{2}{c|}{ Porcentajes } \\
\cline { 3 - 6 } & & Libro 1 & Libro 2 & Libro 1 & Libro 2 \\
\hline \multirow{5}{*}{ Antes: } & Metalectura (M) & 0 & 0 & 0 & 0 \\
Planificación & Observar texto (O) & 6 & 0 & 2,6 & 0 \\
& Estrategia de lectura (E) & 1 & 0 & 0,4 & 0 \\
& Reconocer/aclarar conceptos (AC) & 14 & 3 & 6,1 & 1 \\
& Establecer hipótesis (H) & 15 & 25 & 6,5 & 8,3 \\
& Activar conocimientos previos (ACP) & 12 & 25 & 5,2 & 8,3 \\
\hline & Total actividades planificación & 48 & 53 & 21 & 17,5 \\
\hline \multirow{4}{*}{ Durante: } & Comprobar hipótesis (CH) & 1 & 0 & 0,4 & 0 \\
Supervisión & Contestar preguntas (CP) & 28 & 48 & 12,1 & 15,9 \\
& Subrayar (S) & 1 & 0 & 0,4 & 0 \\
& Vocabulario contextual (VC) & 1 & 0 & 0,4 & 0 \\
\hline \multirow{2}{*}{ Después: } & Total actividades supervisión & 31 & 48 & 13 & 15,9 \\
\hline Evaluación & Contestar preguntas (CP) & 133 & 195 & 57,8 & 64,6 \\
& Resumen/esquema/mapas (R/E/M) & 16 & 3 & 6,9 & 1 \\
& Evaluación estrategia (EE) & 1 & 3 & 0,4 & 1 \\
\hline & Total actividades evaluación & 150 & 201 & 65 & 65,6 \\
\hline & Total & 229 & 302 & 100 & 100 \\
\hline
\end{tabular}

$\mathrm{Al}$ analizar los resultados obtenidos luego de clasificar las actividades respecto del momento de la lectura en el que se enfocan y la estrategia en donde se enmarcan es posible indicar que en el libro 1 en la etapa Antes de la lectura, centrada en la planificación del proceso, se proponen 48 actividades ( $21 \%$ del total), de ellas ninguna apunta a establecer una meta en la lectura (M). Luego se registran seis actividades que contemplan la observación del texto $(\mathrm{O})$ previa la lectura $(12,2 \%)$. Respecto de la estrategia de lectura (E), es posible indicar que una de las actividades se enfoca en esta actividad de planificación (2\%). Respecto de las estrategias orientadas a reconocer o aclarar conceptos (AC) y comprobación de hipótesis $(\mathrm{CH})$ se identificaron 15 actividades $(30,6 \%)$ para cada una de ellas. Por último, relativo a las estrategias orientadas a activar los conocimientos previos (ACP) se encuentran 12 (24,4\%). En cuanto al libro 2, en el momento Antes de la lectura (planificación) se llevan a cabo 53 actividades (17\% del total de actividades), ninguna se enfoca en las estrategias meta de lectura $(\mathrm{M})$, observar texto $(\mathrm{O})$, establecer una estrategia de lectura (EE); solo se identifican tres actividades $(5,7 \%)$ que utilizan la estrategia reconocer/aclarar concepto (AC); 25 actividades utilizan la estrategia establecer hipótesis previa a la lectura $(\mathrm{H})(47,2 \%)$ y, por último, 25 actividades apuntan a activar conocimientos previos (ACP) $(47,2 \%)$.

El momento Durante la lectura (supervisión) en el libro 1 contempla 31 actividades $(14,2 \%)$. De ellas solo el 3,2\% de las actividades corresponden a la comprobación de hipótesis $(\mathrm{CH})$, es decir, una de las actividades se enfoca en emplear este tipo de actividad. La estrategia Contestar preguntas (CP) se observa en 28 de las actividades $(90,3 \%)$. Se utilizan las estrategias subrayado (S) y vocabulario contextual (VC) en una 
actividad para cada una de ellas $(3,2 \%)$. En cuanto al libro 2, se observa que todas las actividades de esta etapa utilizan la estrategia Contestar preguntas (CP) (48 actividades).

En el momento Después de la lectura (evaluación) en el libro 1, los datos indican que 133 de las actividades emplean la estrategia Contestar preguntas (CP) (88,7\%). El uso de resúmenes/esquemas/mapas conceptuales (R/E/M) se observa en 16 actividades $(10,7 \%)$. Por último, una actividad hace mención a la evaluación de la estrategia (EE) de lectura, (0,7\%). Respecto del libro 2, es posible indicar que 195 actividades (97\%) utilizan la estrategia Contestar preguntas $(\mathrm{CP}) ; 3$ actividades $(1,5 \%)$ apuntan a la construcción de resúmenes/esquemas/mapas conceptuales (R/E/M); y 3 actividades $(1,5 \%)$ se dirigen a evaluar la estrategia (EE).

A continuación se resumen los resultados antes expuestos.

Tabla 5. Frecuencia de uso de estrategias metacognitivas según etapa de la lectura por texto

\begin{tabular}{|l|l|r|r|r|c|}
\hline \multirow{2}{*}{$\begin{array}{c}\text { Etapa de } \\
\text { lectura }\end{array}$} & \multicolumn{1}{c|}{ Estrategias } & \multicolumn{2}{c|}{ Frecuencias } & \multicolumn{2}{c|}{ Porcentajes } \\
\cline { 3 - 6 } & & Libro 1 & Libro 2 & Libro 1 & Libro 2 \\
\hline \multirow{5}{*}{ Antes: } & Metalectura (M) & 0 & 0 & 0 & 0 \\
Planificación & Observar texto (O) & 6 & 0 & 12,5 & 0 \\
& Estrategia de lectura (E) & 1 & 0 & 2,1 & 0 \\
& Reconocer/aclarar conceptos (AC) & 14 & 3 & 31,3 & 5,7 \\
& Establecer hipótesis (H) & 15 & 25 & 29,2 & 47,2 \\
& Activar conocimientos previos (ACP) & 12 & 25 & 24,4 & 47,2 \\
\hline & Total actividades planificación & 48 & 53 & 100 & 100 \\
\hline \multirow{5}{*}{ Durante: } & Comprobar hipótesis (CH) & 1 & 0 & 3,2 & 0 \\
Supervisión & Contestar preguntas (CP) & 28 & 48 & 90,3 & 100 \\
& Subrayar (S) & 1 & 0 & 3,2 & 0 \\
& Vocabulario contextual (VC) & 1 & 0 & 3,2 & 0 \\
\hline \multirow{3}{*}{ Después: } & Total actividades supervisión & 31 & 48 & 100 & 100 \\
Evaluación & Contestar preguntas (CP) & 133 & 195 & 88,7 & 97 \\
& Resumen/esquema/mapas (R/E/M) & 16 & 3 & 10,7 & 1,5 \\
& Evaluación estrategia (EE) & 1 & 3 & 0,7 & 1,5 \\
\hline & Total actividades evaluación & 150 & 201 & 100 & 100 \\
\hline & Total & 229 & 302 & & \\
\hline
\end{tabular}

\section{DISCUSIÓN}

Los resultados obtenidos a partir del análisis de ambos libros evidencian que se asigna mayor importancia a la etapa de evaluación del proceso de lectura. Esto implica el desarrollo de un ejercicio centrado en el resultado más que en el proceso. Si consideramos que el enfoque metacognitivo de la comprensión lectora plantea que el proceso de lectura se debe desarrollar considerando tres etapas con actividades definidas en función del objetivo de cada una de ellas (López y Arcienagas, 2004), la propuesta didáctica que se plantea en los libros de Lenguaje y Comunicación de séptimo año básico relega a un segundo 
plano las dos primeras etapas de lectura, centradas en la planificación y la supervisión, enfatizando las actividades de evaluación.

El análisis realizado permitió detectar que las actividades de evaluación están centradas en responder preguntas a partir del texto $(57,8 \%$, libro $1 ; 64,6 \%$, libro 2$)$ en desmedro de las actividades orientadas a producir un texto nuevo a partir de lo leído (resumen) o construir un organizador gráfico (esquemas, mapas conceptuales). Considerando el modelo propuesto en la teoría de la construcción-integración (Kintsch, 1988; Ericsson \& Kintsch, 1995), cuyo último nivel de procesamiento está constituido por la construcción del modelo de situación, la escasa aplicación de estas estrategias (R/M/E) representa una dificultad para desarrollar habilidades de comprensión lectora, porque la elaboración de un texto nuevo o de una forma de representar el contenido del texto da cuenta de este último nivel de procesamiento. De esta forma se está minimizando el desarrollo de actividades orientadas a visualizar el contenido del texto y la relación que se establece entre sus distintos componentes. Considerando que el nivel de representación del modelo situacional es, al parecer, el que mayor importancia tiene para el proceso de comprensión y aprendizaje a partir de un texto, toda vez que en este nivel se generan inferencias y oraciones nuevas que en conjunto constituyen una representación de la situación que el texto describe y pueden soportar reelaboraciones que permitan desarrollar operaciones destinadas al aprendizaje (Gárate et al., 1999), la escasa importancia asignada al desarrollo de habilidades relacionadas con la elaboración de resúmenes, mapas conceptuales o esquemas representaría una falencia del enfoque didáctico planteado en el texto.

La comprensión lectora es un proceso dinámico e interactivo en donde el rol del lector es fundamental, pues este debe controlar y evaluar en forma constante su proceso de comprensión. Desde esta perspectiva resulta relevante el desarrollo de actividades destinadas a evaluar cómo se desarrolló el proceso o qué tan efectiva fue la estrategia empleada. Sin embargo, del universo de actividades analizadas en ambos libros, $0,4 \%$ en el libro 1 y $1 \%$ en el libro 2 , tenían como objetivo desarrollar la conciencia del lector respecto a la aplicación de una estrategia de lectura determinada. Con ello, se observa claramente que las actividades de evaluación desarrolladas en el momento Después de la lectura no tienen como objetivo principal desarrollar un proceso metacognitivo centrado en la evaluación del proceso, sino que dichas actividades se enfocan principalmente en la evaluación de la comprensión del contenido del texto. Relacionando este hallazgo con la teoría planteada por Flavell (Cit. en Osses y Jaramillo, 2008: 191) es posible constatar que el enfoque didáctico que subyace a las actividades de comprensión lectora de los libros analizados se centra en la conciencia acerca del contenido del texto en desmedro de la conciencia respecto de los propios procesos de pensamiento que desarrolla el estudiante.

La comprensión lectora, comprendida como una actividad psicológica compleja, es un proceso que debe estar guiado por un objetivo, a fin de que el lector defina una estrategia en función del mismo (López y Arcienagas, 2004). Este objetivo de lectura se debe explicitar al inicio del proceso, es decir, antes de leer para guiar la lectura; sin embargo, la evidencia obtenida a partir del análisis indica que no se desarrollan actividades orientadas a definir un objetivo de lectura, ya que ninguna actividad considera como estrategia establecer una meta de lectura. Por tanto, en ninguna actividad se insta a definir una estrategia en función del objetivo de lectura (para qué se leerá). Dentro de esta misma etapa resulta relevante también la observación del texto para reconocer el género discursivo al que pertenece, actividad fundamental a la hora de definir una estrategia de 
lectura, recurso escasamente utilizado en el libro 1 (2,6\% de las actividades) y no utilizado en las actividades propuestas en el libro 2. Lo anterior evidencia que la actividad de comprender un texto no se plantea como un proceso en función de un objetivo, por tanto, las actividades no contribuyen a desarrollar la conciencia metacognitiva respecto del proceso que se está realizando.

El momento Durante la lectura, cuyo objetivo es la supervisión del proceso, es el que evidencia menor proporción de actividades: $13 \%$ en el libro 1 y 15,9\% en el libro 2 , Un porcentaje importante de las actividades de esta etapa consiste en plantear preguntas al estudiante a medida que avanza la lectura, esto tendría como objetivo favorecer el monitoreo del proceso de lectura (López y Arcienagas, 2004). Sin embargo, no se entrega una instrucción explícita respecto de esta actividad. En función de lo anterior, es posible señalar que estas actividades no favorecen el desarrollo de la conciencia metacognitiva, pues no se explicita la necesidad de supervisar y controlar el proceso de lectura en función de un objetivo determinado (Osses y Jaramillo, 2008) debido a que tampoco se presentan actividades en función de un objetivo de lectura explícito.

Respecto de la etapa de planificación, ambos libros utilizan con mayor frecuencia las estrategias establecer hipótesis $(\mathrm{H})$ y activar conocimientos previos $(\mathrm{ACP})$ relativos a esta etapa. No obstante, en una etapa posterior no se propone actividad alguna para comprobar la predicción realizada, lo que permite inferir que ambas estrategias se utilizan como un recurso de motivación y contextualización de la lectura y no como una estrategia que requiere de un proceso consciente de comprobación de hipótesis o predicciones.

Durante la etapa de supervisión, el análisis demuestra que no se consideran relevantes las estrategias comprobar hipótesis $(\mathrm{CH})$, subrayar (S) y vocabulario contextual (VC), lo que, en concordancia con lo anteriormente señalado, no promueve la reflexión acerca de lo que el estudiante está leyendo.

En relación con los datos obtenidos tras la comparación de resultados se observa que en ambos libros se enfatiza la etapa de evaluación de la comprensión del texto (mas no la estrategia), manteniendo un alto porcentaje de actividades. La estrategia cuyo uso predomina en esta etapa es Contestar preguntas (CP) acerca del texto $(88,7 \%$ en el libro 1 y $97 \%$ en el libro 2). Respecto del uso de las demás estrategias, se observan diferencias entre ambos libros, porque el libro 1 asigna un mayor porcentaje de uso a la estrategia elaborar resúmenes, esquemas y mapas $(\mathrm{R} / \mathrm{E} / \mathrm{M}), 10 \%$ de las actividades de esta etapa; mientras que en el libro 2 solo se evidencia 1,5\% de las actividades de la etapa. Concerniente a la evaluación de la estrategia (E), ambos libros no asignan importancia a esta actividad. Sin embargo, un porcentaje levemente mayor se observa en el libro 2 (1,5\% libro $2 ; 0,7 \%$, libro 1$)$.

En ambos libros la etapa de planificación ocupa el segundo lugar en el uso de estrategias. Sin embargo, el libro 2 propone actividades de manera más equitativa entre las etapas de planificación y supervisión, lo que permite observar un equilibrio entre estas etapas, a diferencia del libro 1, donde se asigna mayor énfasis a la etapa de planificación. Si bien en ambos libros no se utiliza la estrategia establecer meta de lectura (M), en el libro 2 no se evidencia el uso de las estrategias observar la estructura del texto $(\mathrm{O})$ ni establecer una estrategia de lectura (E). En el libro 1, en cambio, se evidencia su uso aunque en un bajo porcentaje $(12,2 \%$ y $2 \%$ en relación con total de actividades de la etapa). 
En el libro 1 se considera el reconocer o aclarar conceptos (AC) como una estrategia con la misma importancia que establecer hipótesis $(\mathrm{H})$ y activar conocimientos previos (ACP) $(31,3 \%, 29,2 \%$ y $24,4 \%)$ lo que se relaciona con una finalidad de motivación a la lectura más que con un objetivo metacomprensivo. En el libro 2, en cambio, se establece una distribución equilibrada entre las estrategias establecer hipótesis $(\mathrm{H})$ y activar conocimientos previos (ACP) siguiendo la línea de lo anteriormente señalado.

Respecto del momento Durante la lectura, el libro 2 considera solo una estrategia de supervisión (responder preguntas); en cambio en el libro 1 se reconocen las 4 estrategias planteadas en las categorías de análisis, pero con un muy bajo porcentaje $(3,2 \%$, responder preguntas (RP); 3,2\%, subrayar (S); 3,2\% vocabulario contextual (VC) y 90,3\% contestar preguntas (CP)). Estos datos demuestran que ambos libros enfatizan solo el uso de esta última estrategia.

En el libro 2, a pesar de que se evidencia una variedad menor de estrategias en comparación con el libro 1, se observa una sistematización en el uso de las estrategias, lo que podría tener un efecto positivo en los estudiantes, ya que al repetir constantemente una forma de abordar la comprensión lectora se internaliza el uso de las estrategias. Sin embargo, no es posible obviar el hecho de que no se promueve el uso de estrategias metacognitivas para la comprensión de un texto.

Finalmente, considerando el análisis en su totalidad, los datos obtenidos indican que en los libros de Lenguaje y Comunicación de séptimo año básico predomina un modelo didáctico en el que no se estimula el comportamiento estratégico mediante la metacognición, sino que se focaliza en la ejecución de la estrategia de contestar preguntas (CP) al terminar de leer con el fin de evaluar la comprensión, relegando a un segundo plano la evaluación del proceso de lectura y, con ello, el desarrollo de la metacognición.

\section{CONCLUSIÓN}

Esta investigación se ha centrado en el reconocimiento del uso de estrategias metacognitivas en el enfoque didáctico que sustentan las actividades de comprensión lectora. El análisis de los resultados obtenidos permite señalar que los libros de séptimo año básico que con mayor frecuencia se utilizan en nuestro país no abordan las actividades de comprensión lectora incentivando el uso de estrategias metacognitivas que favorezcan el desarrollo de habilidades de comprensión lectora en los estudiantes de séptimo año básico, ya que las actividades se dirigen principalmente a responder cuestionarios acerca de la información entregada en los diversos textos analizados más que a una reflexión relativa a los procesos involucrados en la realización de las tareas de comprensión lectora. Además, se observa que en el desarrollo de las tareas de comprensión lectora no se considera el comportamiento estratégico requerido, el que tiende a posicionar al lector como un sujeto que participa activamente del proceso de lectura planificándolo en función de un objetivo específico, supervisando la aplicación de estrategias determinadas y, finalmente, evaluando tanto la comprensión como las estrategias empleadas.

Los aportes de esta investigación son de carácter práctico, porque permite analizar las estrategias utilizadas por un recurso didáctico que tiene un gran impacto en el sistema educativo chileno, pues corresponden a los textos con los que trabajan la gran mayoría de los establecimientos chilenos. Además, al comparar ambos libros no se encuentran 
diferencias sustanciales entre ambos textos respecto del enfoque metacognitivo con el que se trabajan las actividades; sin embargo se observan diferencias en el abordaje de los contenidos entre ambos libros, debido a que el libro 2 aborda de forma integral las unidades, manteniendo conectadas las actividades que se realizan respecto de la comprensión lectora, los contenidos, la expresión oral y escrita, siendo abordadas por mediodel trabajo de una misma temática, lo que favorece la integración de la información en la memoria de los estudiantes y, con ello, la organización del conocimiento. Además, se observan diferencias en cuanto a la naturaleza de las preguntas que se realizan a los alumnos, estas invitan a los estudiantes a reflexionar respecto de los contenidos abordados, promoviendo el uso sistemático y progresivo de inferencias, proceso cognitivo fundamental en la comprensión lectora.

Frente a lo anterior, es posible que en futuras investigaciones se realice un análisis profundo concerniente al abordaje de los contenidos entre ambos libros y cómo este puede afectar el proceso de enseñanza-aprendizaje de los alumnos y alumnas que lo utilizan. También sería interesante dirigir nuevas investigaciones pertinentes al abordaje que tienen las demás asignaturas con la compresión lectora, si se utiliza un enfoque metacognitivo en la entrega de los contenidos, pues como se ha documentado, es fundamental desarrollar en los estudiantes de nuestro país habilidades que les permitan transformarse en lectores activos, ya que es una herramienta fundamental a la hora de progresar en sus aprendizajes.

Una de las limitaciones de esta investigación está dada por el universo considerado, porque solo se analizó el texto de séptimo año básico, lo que impide generalizar los resultados. Por tanto, se podría pensar que en otros niveles de enseñanza básica es posible que exista otro tratamiento de la comprensión lectora, con énfasis en el componente estratégico y, con ello, en el desarrollo de la metacognición. El desconocimiento de la didáctica que desarrolla el docente en el aula constituye otra limitación de este estudio, pues el profesor, aun cuando no estén explícitas las instrucciones en torno a los momentos de la lectura y sus actividades, puede guiar el proceso de lectura a base de un enfoque metacognitivo. Sin embargo, como en las instrucciones no se explicitan el desarrollo de las tareas de comprensión lectora en función de un comportamiento estratégico, es posible señalar que no constituye una estrategia de trabajo sistematizada y compartida por todos los docentes del sistema escolar chileno.

\section{REFERENCIAS BIBLIOGRÁFICAS}

Baddeley, A. (1986). Working memory. Oxford: Clarendon Press.

Ericsson, K. \& Kintsch, W. (1995). Long-term working memory. Psychological Review, vol. $102(2), 211-245$.

Flavell, J. H. (1976). Metacognitive aspects of problem solving. In L. B. Resnik (Ed.), The nature of intelligence (pp. 231-235). Hillsdale, NJ: Erlbaum.

Gárate, M., Gutiérrez, F., Luque, J., García Madruga, J. y Elosúa, M. (1999). Comprensión lectora y memoria operativa. Barcelona: Paidós.

Karbalaei, A. (2011). Metacognición y comprensión lectora. Íkala. Revista de Lenguaje y Cultura, vol. 16, (28), 5-14.

Kintsch, W. (1998). Comprehension: A paradigm for cognition. Cambridge: Cambridge University Press. 
Welsch, D., Schmalhofer, F. \& Zimny, S. (1990). Sentence memory: A theoretical analysis. Journal of Memory and Language, vol. 29 (2), 133-159.

. (1988). The role of knowledge in discourse comprehension: A construction integration model. Psychological Review, vol. 95 (2), 163-182. vol. 85 (5), 363-394.

. y Van Dijk, T. (1978). Toward a model of text comprehension. Psychological Review,

Lacon, N. y Ortega, S. (2008). Cognición, metacognición y escritura. Revista Signos. Estudios de Lingüística, vol. 41 (27), 231-255.

López, G. y Arcienagas, E. (2004). Metacognición, lectura y construcción del conocimiento. El papel de los sujetos en el aprendizaje significativo. Bogotá: Cátedra UNESCO para la Lectura y la Escritura en América Latina. (2003). El uso de estrategias metacognitivas en la comprensión de textos escritos. Lenguaje, (31), 118-141.

Mason, L., Meadan-Kaplansky, H., Hedin, L. \& Taft, R. (2013). Self-regulating informational text reading comprehension: Perceptions of low-achieving students. Exceptionality, vol. 21 (2), 69-86.

Mcnamara, D. (2004). Aprender del texto: Efectos de la estructura textual y las estrategias del lector. Revista Signos. Estudios de Lingüística, vol. 37 (55), 19-30.

Osses, S. y Jaramillo, S. (2008), Metacognición: Un camino para aprender a aprender. Estudios Pedagógicos, vol. 24 (1), 187-198.

Ozuru, Y., Kurby, C. \& McNamara, D. (2012). The effect of metacomprehension judgment task on comprehension monitoring and metacognitive accuracy. Metacognition and Learning, vol. 7 (2), 113-131.

Pihlainen-Bednarik, K. \& Keinonen, T. (2011). Sixth graders' understanding of their own learning: A case study in environmental education course. International Journal o Environmental \& Science Education, vol. 6 (1), 59-78.

Ray, J. (2011). Análisis de las demandas y mediaciones para la comprensión de textos expositivos en los libros de español y en los libros para el maestro de $2^{\circ}, 3^{\circ}$ y $4^{\circ}$ grados. Revista Mexicana de Investigación Educativa, vol. 16 (49), 415-440.

Yang, K. (2012). Structures of cognitive and metacognitive reading strategy use for reading comprehension of geometry proof. Educational Studies in Mathematics, vol. 80 (3), 307-326.

Vieiro, P. y Gómez, I. (2004). Psicología de la lectura: Procesos, teorías y aplicaciones instruccionales. Madrid: Pearson Educación. 
